\title{
A Review on CBDS in MANET
}

\author{
Navdeep Kaur \\ Computer Science Department \\ MVVE, Jagadhri, India \\ (Received 10 July 2014 Accepted 22 July 2014)
}

\begin{abstract}
Security is an important issue in the integrated MANET-Internet environment because in this environment we have to consider the attacks on Internet connectivity and also on the ad hoc routing protocols. The focus of this work is on different types of attacks on integrated MANET-Internet communication. We consider most common types of attacks on mobile ad hoc networks and on access point through which MANET is connected to the Internet.
\end{abstract}

Index Terms - CBDs, TCP, MANETs, DOS, Sleep Deprivation Attack.

\section{INTRODUCTION}

With the proliferation of mobile technology, the wireless communication is becoming more popular than ever before. This is due to technological advances in laptops \& wireless data communication devices such as wireless MODEM \& Wireless LAN. A Mobile Ad hoc Network (MANET) consists of mobile platforms, which are free to move about arbitrarily. Each of these platforms, herein simply referred to as "nodes", logically consists of a router with possibly multiple IP-addressable hosts and multiple wireless communications devices A node may consist of physically separate networked devices, or may be integrated into a single device such as a Laptop or Personal Computer. The nodes are equipped with wireless transmitters and receivers using antennas which may be Omni-directional (broadcast), highly directional (point-to-point), steerable (arrays) or some combination of them. MANET could be classified under three categories:-

- Internet Based Mobile Ad-hoc Networks (iMANETS) are ad-hoc networks that connects the mobile nodes to the fixed internet gateway nodes.

- Vehicular Ad-hoc Networks (VANET) are used for communication among vehicles and roadside equipments.

- Intelligent Vehicular Ad-hoc Networks (InVANETS) are type of artificial intelligence techniques that help vehicles during collisions, accidents \& etc.

A vulnerability is a weakness in security system. A particular system may be vulnerable to unauthorized data access because the system does not verify a user's identity before allowing data ease to access. MANET is more vulnerable than wired network. Some of the vulnerabilities are as follows:-

1) Lack of centralized management - MANET doesn't have a centralized monitor server. The absence of such management makes the detection of attacks difficult as it is not easy to monitor the traffic in a large scale ad-hoc network. Lack of centralized management will easily break the trust management for nodes.

3) Resource availability - Resource availability is a major issue in MANET. Providing secure communication in such changing environment as well as protection against specific threats and attacks, leads to development of various security architectures which provide safety to the user. Collaborative ad-hoc environments also allow us to develop such security mechanism

3) Scalability - Due to mobile nature of nodes, scale of adhoc network change frequently. So scalability is a major issue concerning security and authentication. Security mechanism should be capable of handling various range and size of network.

\section{LITERATURE OVERVIEW}

In this paper, we have analyzed the security threats an ad-hoc network faces and presented the security objective that need to be achieved. On one hand, the security-sensitive applications of an ad-hoc networks require high degree of security on the other hand, ad-hoc network are inherently vulnerable to security attacks. Therefore, there is a need to make them more secure and robust to adapt to the demanding requirements of these networks.

In [2] authors conclude that the lack of any infrastructure makes the MANET network insecure leading to attacks such as blackhole and grayhole attack. In blackhole attacks, node transmits malicious broadcast informing that it has the shortest path to destination \& attracts all the packets using Forget Route Reply Protocol (RREP) by providing fake information. Author proposes a mechanism called Cooperative Bait Detection Scheme (CBDS) that effectively detects malicious nodes that attempts to launch Collaborative blackhole attacks. Observed results show that DSR (without CBDS) drastically suffers from blackhole attacks when the percentage of malicious nodes increases. This is attributed to the fact that DSR has no secure method for detecting/preventing blackhole attacks. 
Our CBDS scheme shows a higher packet delivery ratio compared with that of DSR. Even in the case where $40 \%$ of the total nodes in the network are malicious, the CBDS scheme still successfully detects those malicious nodes while keeping the packet delivery ratio above $90 \%$.

Cooperative Bait Detection Scheme (CBDS) has been used to tackle blackhole and grayhole attacks caused by malicious nodes [8]. CBDS combines the advantages of both proactive and reactive detection schemes to detect malicious nodes as proactive detection scheme monitors nearby nodes and avoiding attacks in initial stage and reactive detection scheme triggers only when detection node detects significant drop in delivery ratio. It achieves its goal with Reverse tracing technique.

In [1] the author considers the problem of malicious behavior in leader-based MANETs and analyzes solution to the behavior of the leader by means of some checker nodes. Approach presented is vulnerable since a malicious checker can ruin the character of a normal behaving leader, and declare it as a malicious one. The author proposes an efficient self-organizing mechanism which can detect a malicious behaving leader, while protecting a normal behaving leader from being declared as a malicious node. These networks require proper self organizing algorithms to manage themselves effectively. Self-organising algorithms according to tasks are responsible for the solution of management of mobile ad-hoc networks. Best nodes are selected as leader by assigning tasks to them. Leader-based algorithms have large number of applications including routing coordination and cooperative intrusion detection with lots of advantages. Such algorithms should consider presence of misbehaving nodes in network. Misbehavior of nodes could be classified as selfish or malicious behaviors. Selfish nodes misbehave in order to avoid from being selected as leader as they are not interested in serving other nodes. Malicious nodes once selected as leader then launches the Denial of Service (DOS) attack which may lead to problems in network functioning. Malicious behavior is addressed in just a few models. Punish \& Cooperative catch models are proposed in which checker nodes are responsible for monitoring behavior of leader.

\section{Attacks Classification and Routing Protocols in MANET}

\section{Routing Protocols in MANET}

Routing protocols are used in the implementation of routing algorithms to facilitate the exchange of routing information between networks, allowing routers to build routing tables dynamically. In some cases, routing protocols can themselves run over routed protocols: for example, BGP runs over TCP care is taken in the implementation of such systems not to create a circular dependency between the routing and routed protocols .Routing Protocols in MANET are as follows:

1) Proactive MANET protocols: Proactive MANET protocols keeps on updating network topology information constantly ensuring that its available to all the nodes. These protocols reduce network latency and increases data overhead by updating routing information constantly.

2) Reactive MANET protocols: It determines the routing paths only when required. Example of reactive protocol is AODV (Ad-hoc On Demand Distance Vector), DSR (Dynamic Source Routing)

3) Hybrid MANET routing protocols: Hybrid protocols are the integration of both reactive and proactive MANET protocols. There exist a number of routing protocols of globally reactive and locally proactive states. Example of hybrid routing algorithm is Zone Based Routing Protocol (ZRP).

\section{B Attacks in MANET}

An integrated Internet and mobile ad hoc network can be subject to many types of attacks. These attacks can be classified into different categories,

The type of attacks in MANET could be active or passive attacks. The most common security issues are passive attacks that includes information disclosure and eavesdropping whereas active attacks include Denial of service attack, black hole attack \& byzantine attacks. [11]These attacks include injecting erroneous routing information and diverting network traffic through non optimal paths resulting in inefficient routing or creating Routing Loops.

Here our concern is:

This kind of attack is specific to the mobile ad hoc networks. Sleep deprivation attack does not modify or interrupt the existing routing packets in the networks, rather the malicious nodes fabricate their own packets to cause chaos in the network operations. The aim of this attack is to drain off limited resources in the mobile ad hoc nodes (e.g. the battery powers), by constantly makes them busy processing unnecessary packets. Sleep deprivation attack launches message fabrication attacks by injecting huge unnecessary packets into the targeted nodes in the network.

\section{1) Denial of Service (DOS) attack}

A DOS attack [9] may be defined as an event that diminishes or eliminates a network's capacity to perform its expected function. These attacks are launched against server resources or network bandwidth by preventing authorized users from accessing resources. DoS attack may temporarily block service availability or permanently distort information in the network. DoS attacks can exhaust limited wireless resources such as bandwidth, storage space, battery power, CPU, or system memory. Networks are attacked by modifying routing information or changing system configuration, thereby directly attacking data integrity. 


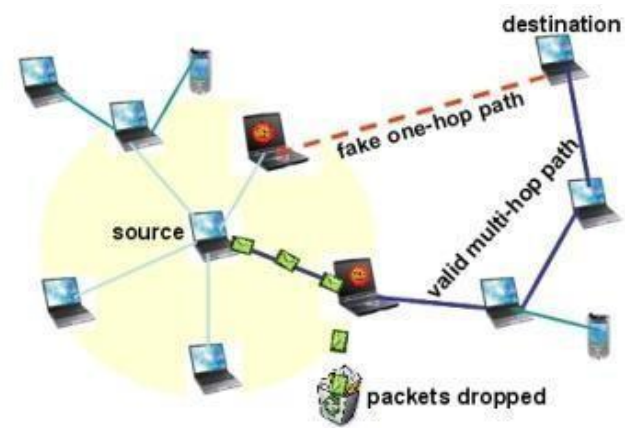

Figure $1:$ dos

In MANETs, nodes act as both routers and ordinary nodes. Dynamic network topology and lack of centralized infrastructure pose a security challenge. Unlike traditional networks, MANETs are more vulnerable to DoS attacks. Limited resources force nodes to be greedy in resource utilization and lack of cooperation between nodes make them so. Misbehave of a small number of nodes renders the whole network inefficient. For instance, a misbehaving node that discards any packets passing through it can result in repeated retransmissions, which in turn cause network congestions. Also, protection of transmitted data in a wireless link remains unguaranteed unlike its wired link counterpart. Hence, any user or receiver within the transmissions range can eavesdrop or interfere with data packets or routing information. Battery power is another critical resource for mobile nodes. If the battery power has been used up due to malicious attacks, the infected node will not be able to provide network services. Sometimes a cracker uses a network of zombie computers to sabotage a specific Web site or server. The idea is pretty simple -- a cracker tells all the computers on his botnet to contact a specific server or Web site repeatedly. The sudden increase in traffic can cause the site to load very slowly for legitimate users. Sometimes the traffic is enough to shut the site down completely. We call this kind of an attack a Distributed Denial of Service (DDoS) attack.

Some particularly tricky botnets use uncorrupted computers as part of the attack. Here's how it works: the cracker sends the command to initiate the attack to his zombie army. Each computer within the army sends an electronic connection request to an innocent computer called a reflector. When the reflector receives the request, it looks like it originates not from the zombies, but from the ultimate victim of the attack. The reflectors send information to the victim system, and eventually the system's performance suffers or it shuts down completely as it is inundated with multiple unsolicited responses from several computers at once.

From the perspective of the victim, it looks like the reflectors attacked the system. From the perspective of the reflectors, it seems like the victimized system requested the packets. The zombie computers remain hidden, and even more out of sight is the cracker himself.

The list of DDoS attack victims includes some pretty major names. Microsoft suffered an attack from a DDoS called MyDoom. Crackers have targeted other major Internet players like Amazon, CNN, Yahoo and eBay. The DDoS names range from mildly amusing to disturbing:

- $\quad$ Ping of Death - bots create huge electronic packets and sends them on to victims

- Mailbomb - bots send a massive amount of e-mail, crashing e-mail servers

- Smurf Attack - bots send Internet Control Message Protocol (ICMP) messages to reflectors, see above illustration

- Teardrop - bots send pieces of an illegitimate packet; the victim system tries to recombine the pieces into a packet and crashes as a result

Once an army begins a DDoS attack against a victim system, there are few things the system administrator can do to prevent catastrophe. He could choose to limit the amount of traffic allowed on his server, but this restricts legitimate Internet connections and zombies alike. If the administrator can determine the origin of the attacks, he can filter the traffic. Unfortunately, since many zombie computers disguise (or spoof) their addresses, this isn't always easy to do.[12]

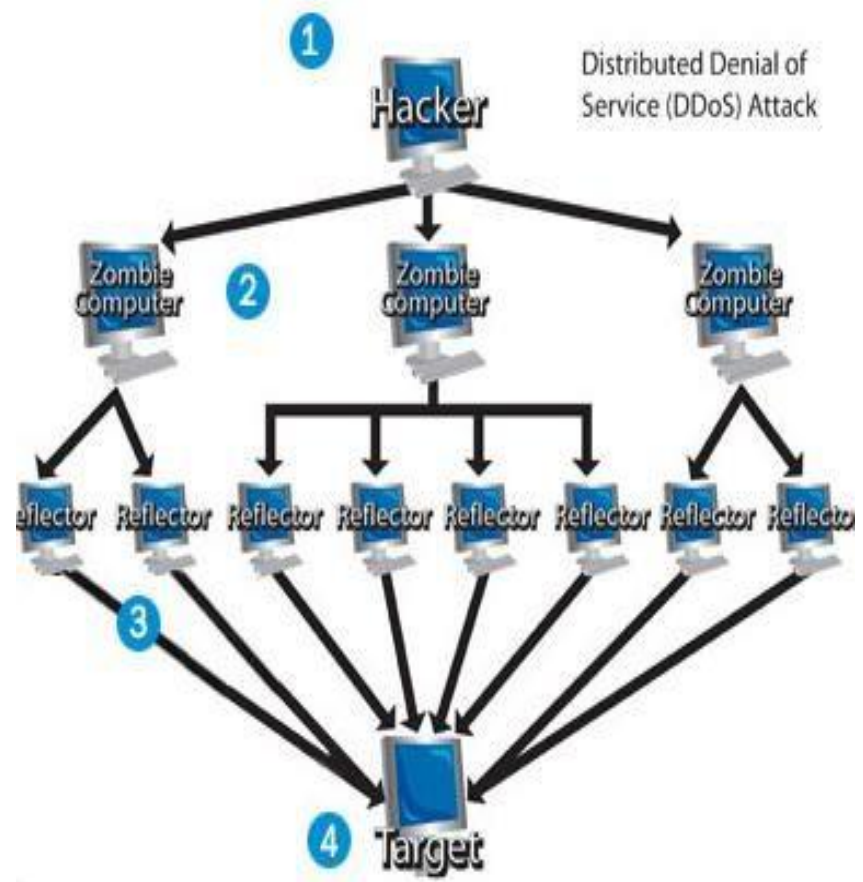

Figure 2 : Denial of service

\section{2) Sleep Deprivation attack}

This kind of attack is specific to the mobile ad hoc networks. Sleep deprivation attack does not modify or interrupt the existing routing packets in the networks, rather the malicious nodes fabricate their own packets to cause chaos in the network operations. The aim of this attack is to drain off limited resources in the mobile ad hoc nodes (e.g. the battery powers), by constantly makes them busy processing unnecessary packets. Sleep deprivation attack launches message fabrication attacks by injecting huge unnecessary packets into the targeted nodes in the network. For example, a node in the network is flooded by the attacker by sending a 
huge number of route request (RREQ), route replies (RREP) or route error (RERR) packets to the targeted node. The result of this injection is that the particular node is unable to participate in the routing mechanisms and rendered unreachable by the other nodes in the networks thus reducing the number of contact points inside network. Also, such processing drains the battery power of the target node.

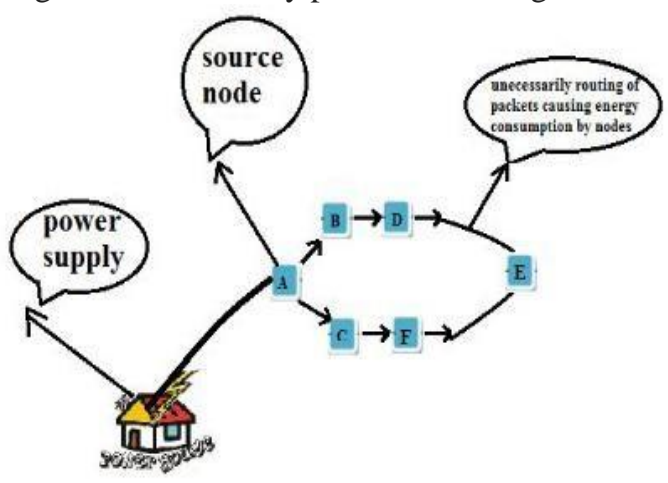

Figure 3 : Sleep deprivation attack

\section{CONCLUSION}

In this paper, we have analyzed the security threats an ad-hoc network faces and presented the security objective that need to be achieved. We have studied different types of attack .ie. DOS Attack and Sleep Derivation Attack. On one hand, the security-sensitive applications of an ad-hoc networks require high degree of security on the other hand, ad-hoc network are inherently vulnerable to security attacks. Therefore, there is a need to make them more secure and robust to adapt to the demanding requirements of these networks.

\section{FUTURE SCOPE}

There is a need for standardized, secure, and interoperable routing and interface solution(s) for mobile networking support. The future holds the possibility for deploying inexpensive, IP internetworking compatible solutions to form self-organizing, wireless routing fabrics for commercial and military use. In future work, CBDS can be deployed on various other security threats in MANET and it results could be checked for validation.

\section{References}

[1] Chin-Feng Lai, Han-Chieh Chao, Jian-Ming Chang, Isaac Woungang, and Po-Chun Tsou, Member, IEEE.Defending Against Collaborative Attacks by Malicious Nodes in MANETs: A Cooperative Bait Detection Approach.

[2] S. M. A. Pari, M. Noormohammadpour, M. J. Salehi, B. H. Khalaj, H. Bagheri and M. Katz, "A self-organizing approach to malicious detection in leader-based mobile ad-hoc networks," 2013 IFIP Wireless Days (WD), Valencia, 2013, pp. 1-3. doi: 10.1109/WD.2013.6686475
[3] Rakesh kumar Sahu,Narendra S chaudhari "performance evaluation of ad hoc network underblack hole attack 978-14673-4805-8/\$31.00,IEEE 2012

[4] Richard Yu, Helen Tang, Minyi Huang and Yanwei Wang, Member, IEEE. A Mean Field Game Theoretic Approach for Security Enhancements in Mobile Ad hoc Networks.

[5] Durgesh Kumar Mishra (Acropolis Institute of Technology and Research, Indore, India). Mahakal Singh Chandel (Arjun Institute of Advaced Studies and Research Centre, Indore, India), Rashid Sheikh. Security Issues in MANET: A Review. [6] Li Shi-Chang, Yang Hao-Lan, Zhu Qing-Sheng College of Computer Science Chongqing University Chongqing, China. Research on MANET Security Architecture design.

[7] Luis Javier García Villalba , Julián García Matesanz , Ana Lucila Sandoval Orozco 1,3 and José Duván Márquez Díaz,"Auto-Configuration Protocols in Mobile Ad Hoc Networks", The 11th International Workshop on Knowledge Management and Acquisition for Smart Systems and Services (PKAW 2010)

[8] S. M. A. Pari, M. Noormohammadpour, M. J. Salehi, B. H. Khalaj, H. Bagheri and M. Katz, "A self-organizing approach to malicious detection in leader-based mobile ad-hoc networks," 2013 IFIP Wireless Days (WD), Valencia, 2013, pp. 1-3. doi: 10.1109/WD.2013.6686475 